\title{
Spreadsheets: A Tool for e-Learning - A Case of Matrices in Microsoft Excel
}

\author{
Nchimunya Chaamwe and Langstone Shumba
}

\begin{abstract}
Spreadsheets made their first appearance for personal computers in 1979 in the form of VisiCalc, an application designed to help with accounting tasks. Over the years the application area for spreadsheets has multiplied. One application that is becoming more pronounced is in the teaching and learning of pupils and students. A Spreadsheet can be used as a powerful learning tool for both primary and secondary school pupils. Spreadsheets offer concrete ways to explore abstract concepts in mathematics and other subjects.

Mathematical ideas are conveyed in several different ways using pictures, colored squares, and number lines. A spreadsheet can be a helpful tool for visual learning. Using spreadsheets also promotes higher order thinking skills.

Spreadsheets contain a variety of formulas, which can be used in teaching mathematics. These formulas can be generalized, so that students can see how the outcome is changed when one of the variables in the formula is altered. The spreadsheet tool also promotes the development of problem solving skills and supports "What if..." type questions. Apart from using ready-made formulas pupils can make up their own formulas to manipulate numbers. This paper proposes the use of Ms Excel in the teaching and learning of Algebra in secondary schools. The paper gives a specific example on how a topic such as matrices can be delivered using Ms excel.
\end{abstract}

Index Terms-Algebra, education, matrices, Microsoft Excel (Ms Excel), spreadsheets.

\section{INTRODUCTION}

Recent trends in Teacher education have emphasized the importance of teaching and learning with technology rather than learning about technology. This implies that teachers should learn to use ICT related technology as a cognitive tool to enhance pupil or student learning of content material other than acquiring isolated skills in basic computation application or merely learning a specific programming language [1]. To promote this usage of Technology for pupils or students conceptual development, Teachers should learn how to use readily available software like spreadsheets as a tool for E-learning purposes.

E-learning can be defined as the delivery of a learning, training or education program by electronic means. E-learning involves the use of a computer or electronic device (e.g. a mobile phone) in some way to provide training, educational or learning material [2].

A spreadsheet can be defined as an electronic worksheet used to organize and manipulate numbers and display options for what-if analysis. The basic construct of an electronic

Manuscript received September 24, 2014; revised January 23, 2015.

The authors are with the Copperbelt University, Zambia (e-mail: nchimunya2009@gmail.com). spreadsheet is an array of rows and columns with automatic update and display of results. This paradigm has been extended with libraries of mathematical and statistical functions, versatile graphing and charting facilities, powerful add-ins such as Microsoft Excel's Solver, attractive and highly functional graphical user interfaces, and the ability to write custom code in languages such as Microsoft's Visual Basic for applications [3]. Users can manipulate numbers by using stored formulas and calculate different outcomes and this capability makes a spreadsheet an ideal tool for the teaching and learning of mathematics. Popular electronic spreadsheet packages include Lotus 1-2-3, Quattro Pro, and Ms Excel. The first electronic spreadsheet though was VisiCalc which appeared in 1979. It was created by Dan Bricklin and Bob Frankston for the Apple II platform. The creators later sold the rights in VisiCalc to Lotus Development Corporation, which developed Lotus 1-2-3 for the IBM PC in 1982. Then came Microsoft Excel, which was developed first for the Apple Macintosh, but which was also the first real Microsoft Windows application [3]-[5].

The use of spreadsheets allows pupils or students to explore solution alternative processes that go beyond symbolic manipulations and provide students with a deeper understanding of concepts embedded in a problem. One unique use of spreadsheets is the ability to interactively model and simulate mathematical situations. Mathematics teachers can use spreadsheets to create experimental environments for discovering mathematical relationships. These experiments can assist pupils or students in their conceptualization of relationships among numerical, graphical and algebraic representations [6].

This paper particularly investigates Ms Excel as a tool for the teaching and learning of mathematics. The paper demonstrates how matrices can be manipulated using excel.

The rest of the paper is organized as follows: Section II reviews literature on similar work, Section III gives the demonstration of how excel can be used to teach matrices and Section IV demonstrates the solving of systems of equation using matrices in Ms excel and Section V concludes the paper.

\section{LITERATURE REVIEW}

ICTs and particularly spreadsheets in teaching mathematics has increased significantly in recent years. Spreadsheets can be used to implement mathematical algorithms, models, visualizations, and applications naturally and effectively through interactive constructions and creative graphical displays [7]. Scholars have studied the impact of integrating ICTs in the teaching and learning of mathematics. Studies on 
the teaching and learning of mathematics using spreadsheets have also been done. This section discusses and analyses these studies as they relate to this paper.

A study was done to investigate the effect of integrating Microsoft Excel into a high school algebra class. The results from the study indicated a slight increase in student achievement when Excel was used. A teacher-created final exam and two Criterion Referenced Tests were used to measure the level of success. One of the Criterion Referenced Tests indicated that the variability of the students scores were reduced considerably in the class using Excel, indicating that possibly an interest in the course was generated and that student' interests were spurred by the use of the software [8].

Another study was done which looked at the learning activity in middle school which made use of a spreadsheet to mediate students' construction of meanings for statistical conceptions. The study illustrated the integration of computers in teaching and learning statistics as it brings about a cascade of changes in curriculum materials, classroom praxis, and students' ways of learning. The study also looked at the impact of technological tools on teaching and learning statistics by emphasizing how the computer lends itself to supporting cognitive and sociocultural processes. The authors concluded that the use of spreadsheets in the teaching of statistical concepts enhances the understanding of these concepts by students [9].

A further study was done that looked at the use of Excel in the introductory course in statistics in a business school. An attempt was made to identify how business school programs differ, and how the use of Excel to work cases or examples can contribute to a business statistics course that is in keeping with the schools and the instructor's learning objectives. The study presented some ideas for using spreadsheets that may be appropriate in teaching statistics in the business school. The study concluded that the use of Excel together with realistic business examples and exercises can contribute greatly to students understanding of both business and statistics [10].

A study was carried out study that included the demonstration of techniques that enable educators to design animated graphical displays in their spreadsheet constructions in order to produce powerful classroom demonstrations to enhance mathematical understanding. The study demonstrated how Ms Excel can be used to create animated and interactive graphs of functions [7].

A review that was done on the using of spreadsheets in the learning and teaching of mathematics suggested that one way that seems to help pupils move from a non-algebraic to an algebraic approach is through work with spreadsheets. The paper argued that, in using such a tool, compared to using paper and pencil, pupils appear to be able to learn more readily to express general mathematical relationships using the symbolic language in the spreadsheet environment which is a key to making progress in algebra. The review suggested that the other teaching advantages of spreadsheets are that pupils are likely to be relatively familiar with them as these are used in other lessons in school and that pupils are also likely to have access to spreadsheet software outside of school, including at home [11].

Ref. [12] in her paper challenges mathematics teachers to rethink the mathematics they teach and investigate technological tools for learning mathematics, and consider how they can support students in learning mathematics with technology as a tool. The author suggest that mathematics teachers must redesign their curriculum and instruction to help students learn about the technology they will use to learn mathematics and one such tool is a spreadsheet. Mathematics teachers are challenged to think about scaffolding students learning about spreadsheets while they are also learning mathematics.

The reviewed research and other researches seem to agree on one point that spreadsheets can enhance the teaching, learning and understanding of mathematics in both primary and secondary school. Ref. [3] argues that there is no longer a need to question the potential for spreadsheets to enhance the quality and experience of learning that is offered to students. Traditional barriers (particularly the lack of facilities to use spreadsheets in assessment contexts) need to be removed, either by ensuring that access to computers is improved or by changing assessment methods. Further expansion is needed of the types of topics that can be effectively covered by spreadsheet examples. This paper therefore will aim at highlighting a few examples of using spreadsheets by teachers to teach algebra and specifically matrices in class.

\section{SOLVING MATRICES IN MS EXCEL}

The main purpose of this chapter is to show how to work with matrices in Excel and use matrices to solve linear systems of equations

\section{A. Matrices and Matrix Operations}

This section shows how matrices can be represented in Excel. A matrix may be thought of as a two-dimensional table. Fig. 1 below shows how matrices $\mathrm{A}, \mathrm{B}$ and $\mathrm{C}$ may be represented in a spreadsheet. We will adopt a convention that a matrix is enclosed with lines on the left and right hand sides.

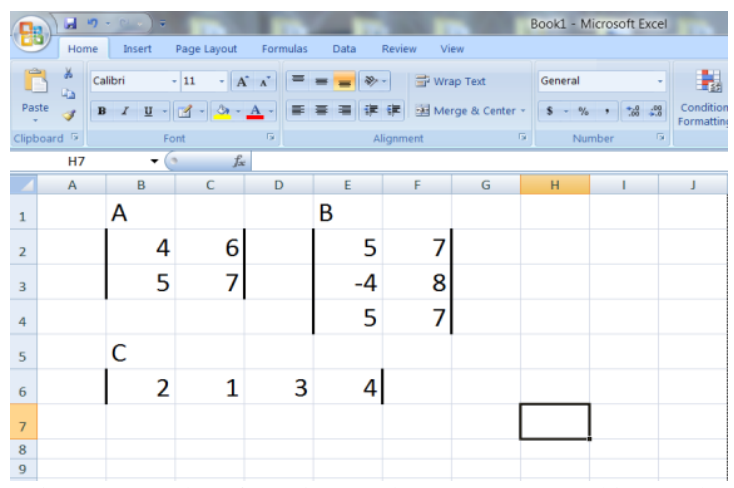

Fig. 1. Examples of matrices as they are represented in Excel.

When working with matrices in Excel, special array formulas and array functions are used. There are special rules that apply to these functions. The reason for this is that array functions return arrays. For example, adding two $m \times n$ matrices the result is an $m \times n$ matrix. When entering the formulas for solving matrices we must take this into account. We do this by entering the formula for solving matrices directly into a matrix.

\section{B. Matrix Addition}

As an example, consider the following addition of matrices. 


$$
\left[\begin{array}{ll}
4 & 6 \\
6 & 7
\end{array}\right]+\left[\begin{array}{ll}
4 & 5 \\
4 & 3
\end{array}\right]
$$

In order to perform this summation, Write a formula that adds the two ranges. Write $=\mathrm{C} 3: \mathrm{D} 4+\mathrm{G} 3: \mathrm{H} 4$ directly. Do not press <Enter> at this point and the spreadsheet should look something like Fig. 2. Note that the entire range C7:D8 is selected.

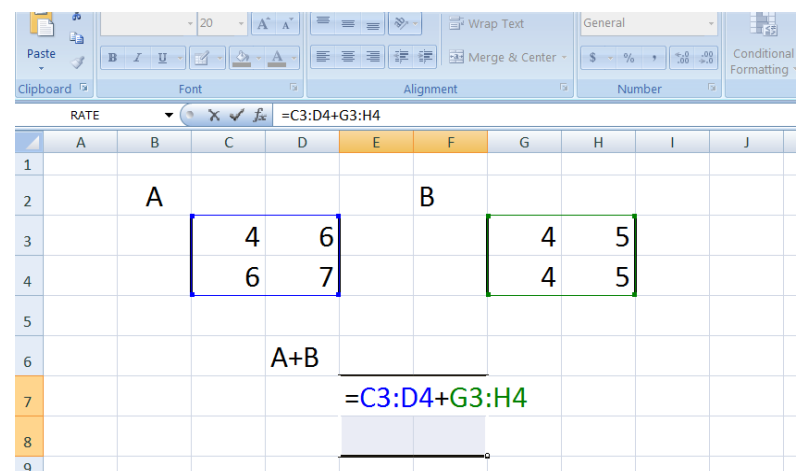

Fig. 2. Showing the addition formula and the selected range.

Then press $\langle$ Ctrl $>+\langle$ Shift $>+\langle$ Enter $>$ and the spreadsheet will look something like in Fig. 3.

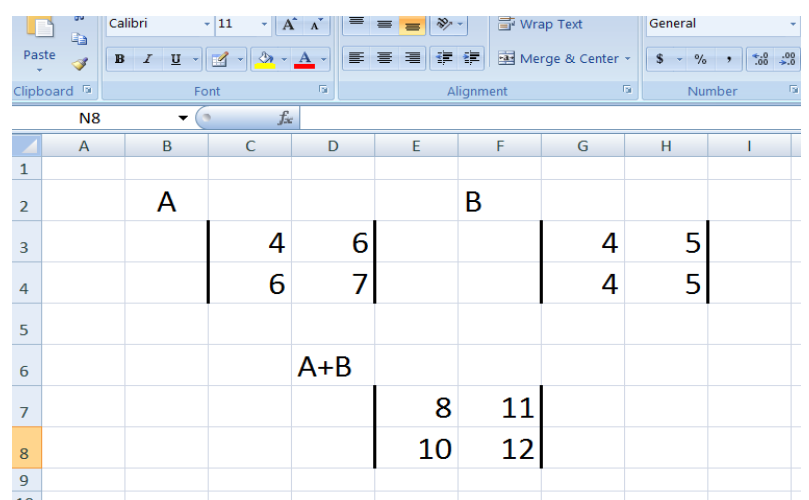

Fig. 3. Showing the final matrix addition answer.

\section{Matrix Scalar Multiplication}

We can multiply any matrix with a scalar. As an example consider the following scalar multiplication of a matrix:

$$
4 \times\left[\begin{array}{ll}
3 & 4 \\
4 & 2
\end{array}\right] .
$$

In order to multiply a matrix by a scalar, write $=4 * \mathrm{C} 3$ :D4 . The spreadsheet should look like Fig. 4.

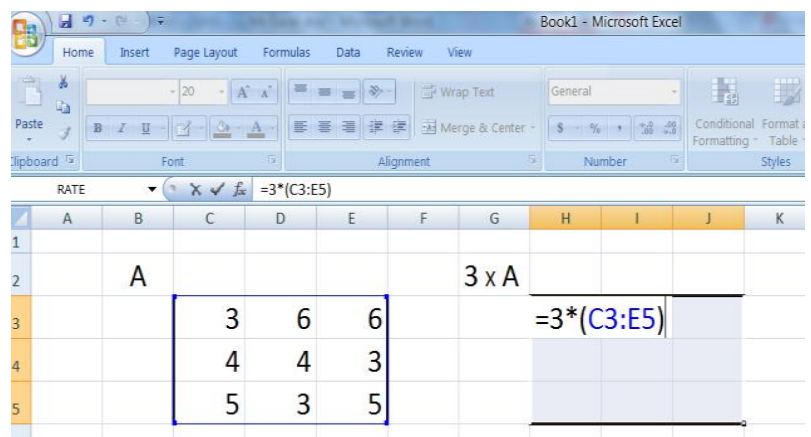

Fig. 4. Showing the scalar multiplication formular and the selected range.
Then press $\langle$ Ctrl $>+\langle$ Shift $>+\langle$ Enter $>$ the spreadsheet will look something like in Fig. 5.

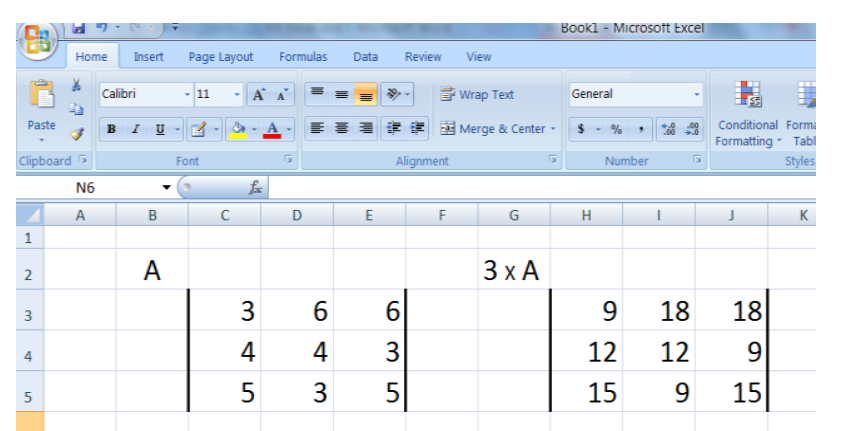

Fig. 5. The spreadsheet showing the answer after scalar multiplication.

\section{Matrix Multiplication}

To perform matrix multiplication's in Excel, we must use the function $=$ MMULT $(\arg 1, \arg 2)$. This function takes two matrices as arguments and returns their product. As an example consider the following multiplication of matrices.

$$
\left[\begin{array}{lll}
3 & 4 & 5 \\
2 & 3 & 3 \\
3 & 2 & 2
\end{array}\right] \times\left[\begin{array}{lll}
2 & 2 & 2 \\
4 & 3 & 3 \\
2 & 2 & 3
\end{array}\right] .
$$

In order to multiply the two, rite=MMULT(C3:E5,H3:J5) directly in the spreadsheet and it will look something like Fig. 6.

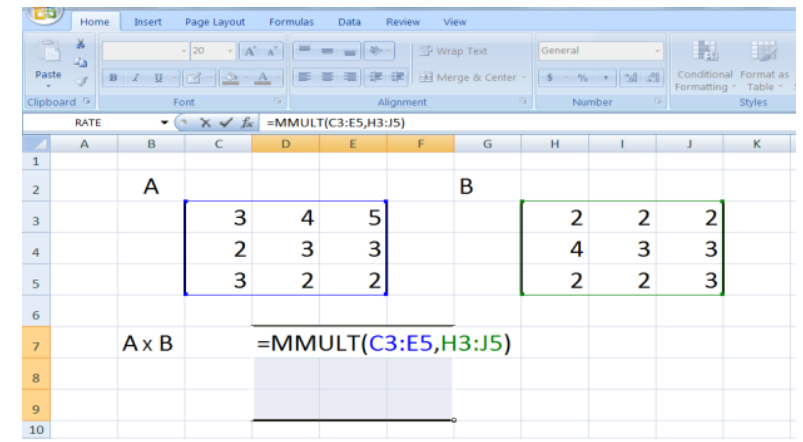

Fig. 6. Showing the matrix multiplication formular and selected range.

After pressing $\langle$ Ctrl $>+<$ Shift $\rangle+<$ Enter $>$ the spreadsheet will look something like in Fig. 7.

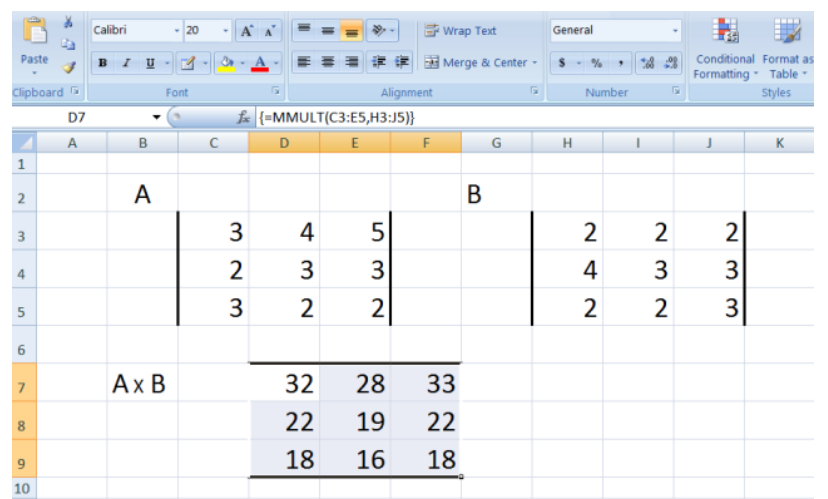

Fig. 7. Showing the final answer for matrix multiplication.

When using the MMULT function you must make sure that 
when you multiply an $m \times n$ matrix with a $k \times h$ matrix, $\mathrm{n}$ must equal $\mathrm{k}$. If not, the result will give a \#VALUE message which means that the input is not correct. It must be taken care that the cells where the answer will be entered is an $m \times h$ matrix.

\section{E. The Transpose of a Matrix}

Excel has a function for transposing matrices. The syntax of this function is =TRANSPOSE( $\arg$ ). To transpose an $\mathrm{m} \mathrm{x} \mathrm{n}$ matrix you must select an $\mathrm{n} x \mathrm{~m}$ matrix to enter the results in. As an example consider the following matrix.

$$
\left[\begin{array}{llll}
3 & 4 & 4 & 5 \\
2 & 3 & 2 & 4 \\
2 & 2 & 1 & 5
\end{array}\right] .
$$

In order to transpose a matrix in excel, write $=$ TRANSPOSE(C3:F5) directly in the spreadsheet and it will look something like Fig. 8.

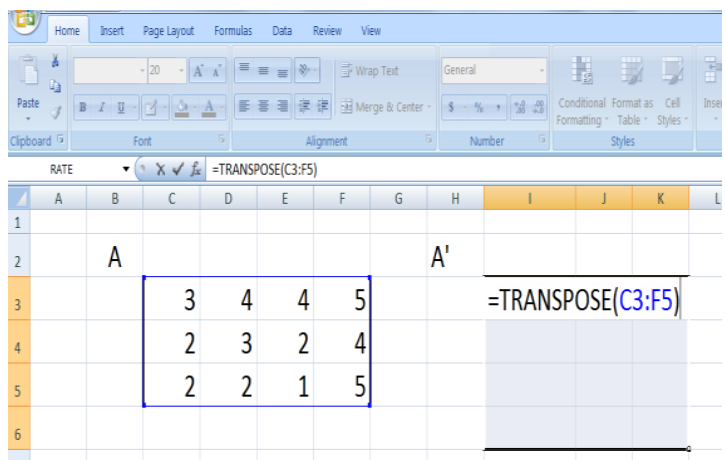

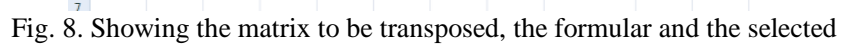
range.

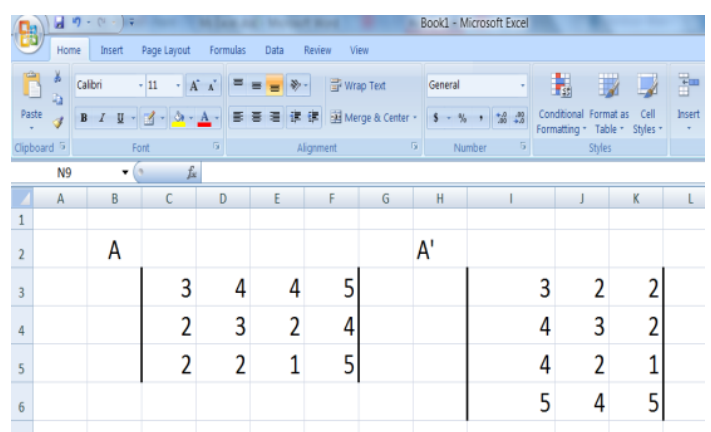

Fig. 9. Showing the final answer after transposing the matrix.

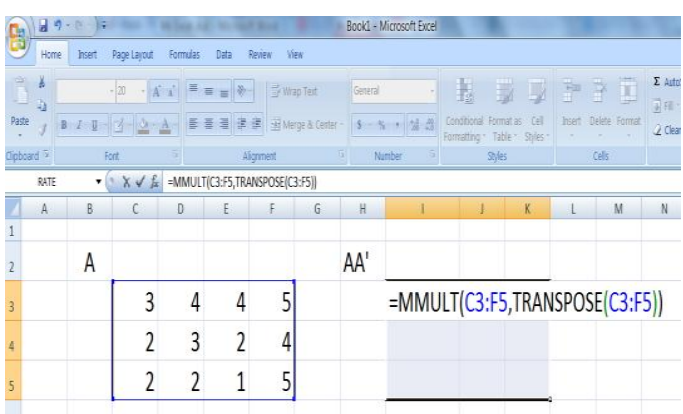

Fig. 10. Showing the multiplication of a matrix with its transpose formular and the selected range.

After pressing $<\mathrm{Ctrl}>+<$ Shift $>+<$ Enter $>$ the spreadsheet will look something like in Fig. 9.
A matrix can be multiplied with its transpose by combining the =MMULT command and the =TRANSPOSE command. If matrix $\mathrm{A}$ is in the cell range $\mathrm{C} 3: \mathrm{F} 5, \mathrm{AA}^{\prime}$ can be calculated by entering the formula $=$ MMULT $(\mathrm{C} 3: \mathrm{F} 5$, transpose $(\mathrm{C} 3: \mathrm{F} 5))$ into a $3 \times 3$ matrix.

A'A can be calculated by entering =MMULT (TRANSPOSE(C3:F5),C3:F5) into a 4x4 matrix. As an example consider the following matrix

$$
\left[\begin{array}{llll}
3 & 4 & 4 & 5 \\
2 & 3 & 2 & 4 \\
2 & 2 & 1 & 5
\end{array}\right] .
$$

In order to multiply a matrix with its transpose, write $=$ MMULT $(\mathrm{C} 3: \mathrm{F} 5$, transpose $(\mathrm{C} 3: \mathrm{F} 5)$ directly the spreadsheet will look something like Fig. 10.

After pressing $<$ Ctrl $>+<$ Shift $>+<$ Enter $>$ the spreadsheet will look something like in Fig. 11.

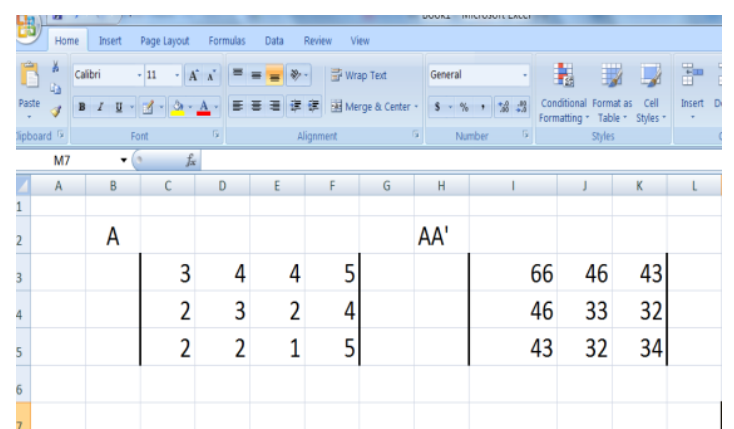

Fig. 11. Showing the final answer after the multiplication of a matrix with its transpose.

\section{F. Determinant of a Matrix}

To find the determinant of a matrix in Excel, we must use the function =MDETERM(arg). Note that the determinant of a matrix is a scalar. To find the determinant of an $\mathrm{m} \mathrm{x} \mathrm{n}$ matrix you must select a single cell to enter the results in. As an example consider the following matrix.

$$
\left[\begin{array}{llll}
3 & 4 & 4 & 5 \\
2 & 3 & 2 & 4 \\
2 & 4 & 2 & 2 \\
1 & 2 & 1 & 5
\end{array}\right]
$$

In order to find the determinant of a matrix, write $=$ MDETERM(C3:F6) directly and the spreadsheet will look something like Fig. 12.

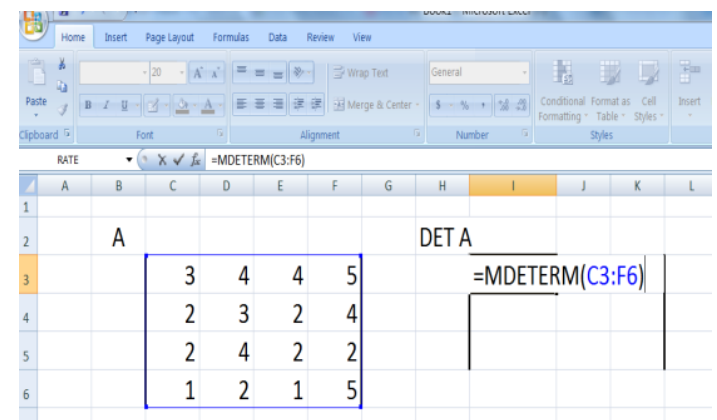

Fig. 12. Showing the formular for finding the detrminant of a matrix and the selected range. 
After pressing $\langle$ Ctrl $>+<$ Shift $>+<$ Enter $>$ the spreadsheet will look something like in Fig. 13.

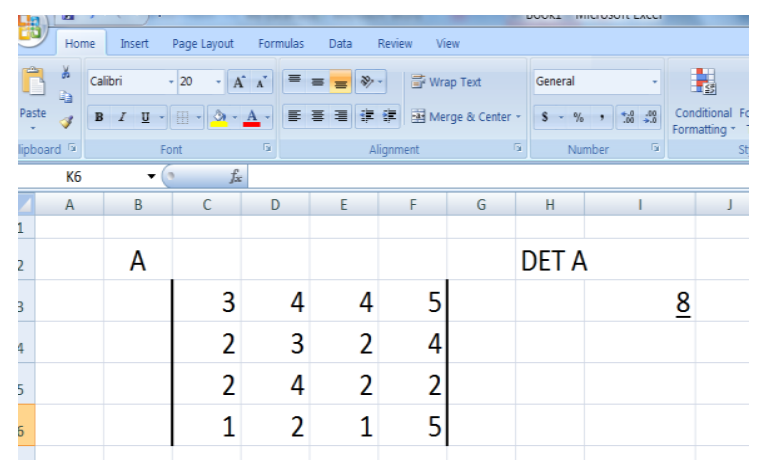

Fig. 13. Showing the derteminant of a matrix.

\section{G. The Inverse of a Matrix}

The inverse of a matrix can be found in Ms excel by using the function =MINVERSE $(\arg )$ function. To find the inverse of a $\mathrm{m} \times \mathrm{n}$ matrix, select $\mathrm{m} \times \mathrm{n}$ cells to enter the results in. As an example consider the following matrix

$$
\left[\begin{array}{llll}
3 & 4 & 4 & 5 \\
2 & 3 & 2 & 4 \\
2 & 4 & 2 & 2 \\
1 & 2 & 1 & 5
\end{array}\right]
$$

In order to find the inverse of this matrix, write $=$ MINVERSE(C3:F6) directly and the spreadsheet will look something like Fig. 14.

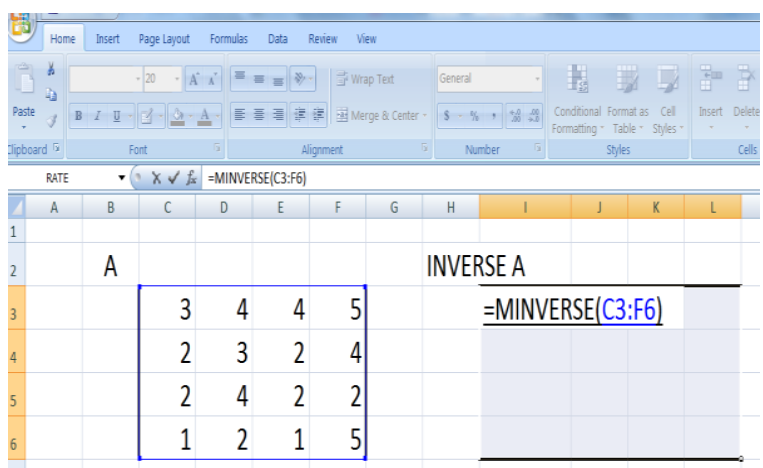

Fig. 14. Showing the formular for finding the invesre of a matrix and the selected range.

After pressing $\langle$ Ctrl $>+<$ Shift $>+<$ Enter $>$ the spreadsheet will look something like in Fig. 15.

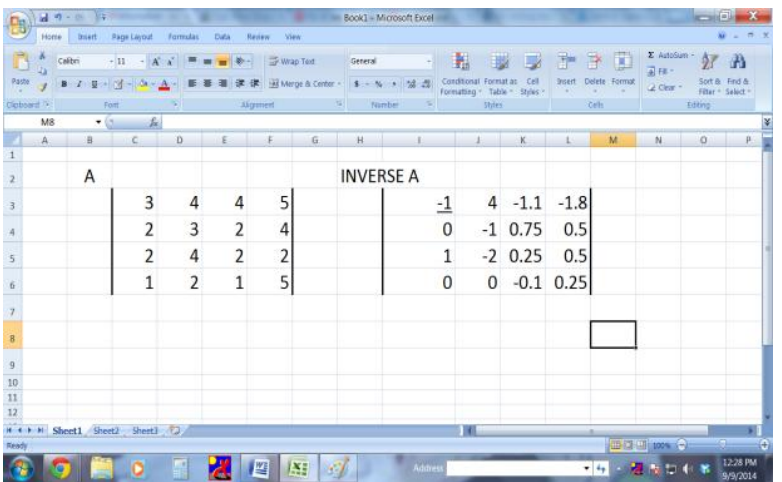

Fig. 15. Showing the inverse of a matrix.

\section{USING MATRICES TO SOLVE SYSTEMS OF EQUATIONS IN} EXCEL

\section{A. Using the Inverse of a Matrix}

As an Example, Solve the following Systems of Linear Equations using the inverse of a matrix:

$$
\begin{aligned}
& x+y+z=6 \\
& 2 y+5 z=-4 \\
& 2 x+5 y-z=27
\end{aligned}
$$

The equations could be written in form of a matrices as:

$$
\left[\begin{array}{rrr}
1 & 1 & 1 \\
0 & 2 & 5 \\
2 & 5 & -1
\end{array}\right]+\left[\begin{array}{l}
x \\
y \\
y
\end{array}\right]=\left[\begin{array}{l}
6 \\
-4 \\
27
\end{array}\right]
$$

We can call the matrices "A", "X" and "B" respectively and the equation becomes:

$$
\mathrm{AX}=\mathrm{B}
$$

Then the solution is $\mathrm{X}=\mathrm{A}-1 \mathrm{~B}$

We can find $\mathrm{X}$ by using matrix multiplication and the Inverse function in Excel. In order to carry out this multiplication in excel, write = MMULT((MINVERSE(C3:E5),H3:H5)) directly and the spreadsheet will look something like Fig. 16.

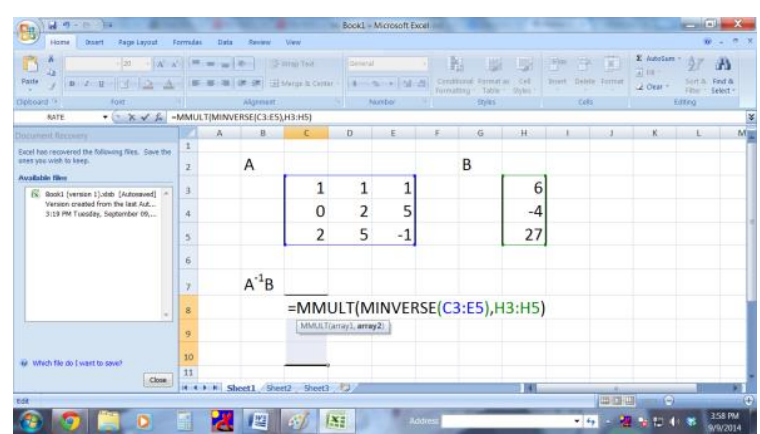

Fig. 16. Showing the formular for multiplying a matrix and the inverse of a second matrix.

After pressing $\langle\mathrm{Ctrl}>+<$ Shift $>+<$ Enter $>$ the spreadsheet will give the solution of $\mathrm{A}^{-1} \mathrm{~B}$ as shown in Fig. 17 .

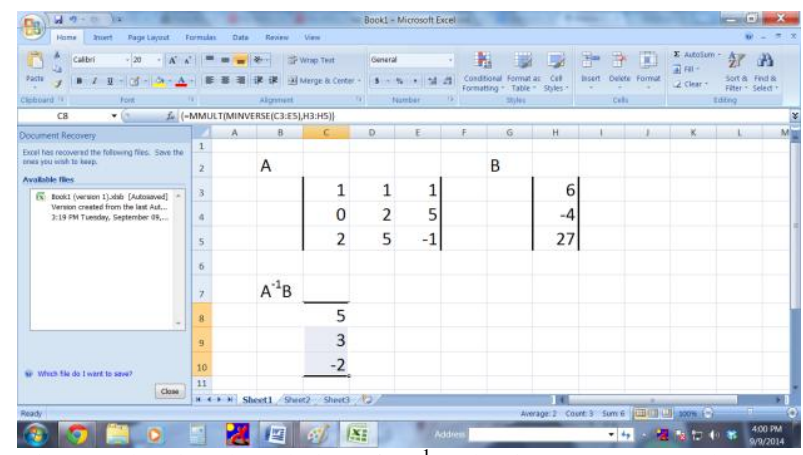

Fig. 17. Showing the solution of $A^{-1} B$, that is $x=5, y=3$ and $z=-2$.

\section{B. Using Determinants to Solve Systems of Equations: Cramer's Rule}

Given the following general systems of equations: 


$$
\begin{aligned}
& a_{1} x+b_{1} y+\mathrm{c}_{1} z=d_{1} \\
& a_{2} x+b_{2} y+\mathrm{c}_{2} z=d_{2} \\
& a_{3} x+\mathrm{b}_{3} y+\mathrm{c}_{3} z=\mathrm{d}_{3}
\end{aligned}
$$

The solution of three linear equations in three unknowns is given by

$$
x=\frac{\Delta x}{\Delta}, y=\frac{\Delta y}{\Delta}, \quad z=\frac{\Delta z}{\Delta}
$$

where $\Delta$ is the determinant of the coefficients $\mathrm{x}, \mathrm{y}$ and $\mathrm{z}$.

And $\Delta x, \Delta y, \Delta z$ are determinants in which the column of $x$ - coefficients, $y$ - coefficients and $z$-coefficients are replaced by the column constants respectively.

We can find $\mathrm{x}, \mathrm{y}$ and $\mathrm{z}$ by using determinant function in Excel. In order to determine these values in excel, write $=$ MDDETERM(I2:K4)/MDETERM(C2:E4) directly and the spreadsheet will look something like Fig. 18.

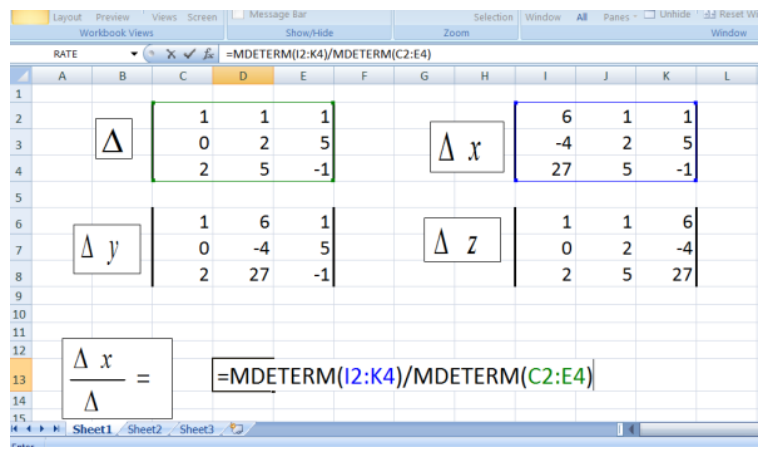

Fig. 18. Showing the formular for solving systems of equations using cramers rule.

After pressing $<\mathrm{Ctrl}>+<$ Shift $>+<$ Enter $>$ the spreadsheet will give the solutions as shown in Fig. 19.

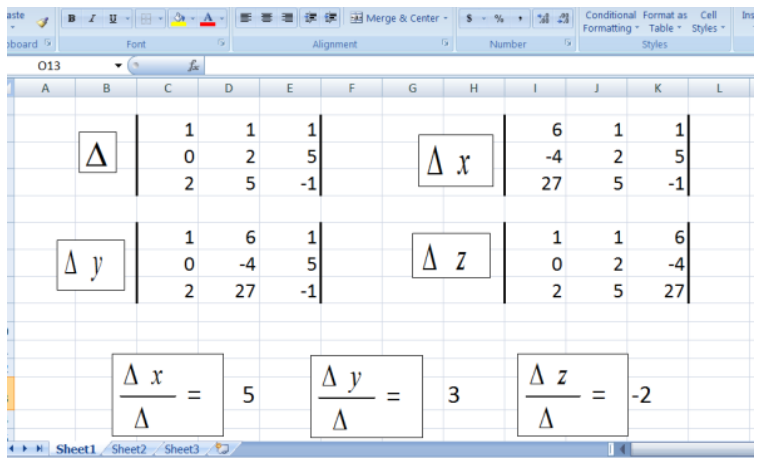

Fig. 19. The spreadsheet showing the solution of systems of equations using cramers rule, that is $x=5, y=3$ and $z=-2$.

\section{CONCLUSION}

A Researchers world over have agreed that the use of technology enhances the understanding of mathematics and other similar subjects by pupils and students primary and Secondary schools. There are a number of software tools that can be used for this purpose on the market. One such tool which is usually overlooked by most educators is Microsoft Excel. Ms Excel has an advantage over other tools in that it is widely available and relatively easy to use. This paper demonstrated how Ms Excel can be used to teach Matrices and their use in solving systems of equations.

\section{REFERENCES}

[1] S. Abramovich and H. S. Drier, "Preparing K-12 mathematics teachers to use a spreadsheet as an instructional tool, in Proc. from the International Meeting of the Society for Informational Technology and Teacher Education, pp. 950-954, San Antonio Texas, 1999.

[2] D. Stockley. (2003). E-learning definition and explanation. [Online]. Available: http://www.derekstockley.com.au/elearn.html

[3] J. Baker and S. J. Sugden, "Spreadsheets in education - The first 25 years," Spreadsheets in Education (eJSiE), vol. 1, no. 1, 2007.

[4] D. Bricklin. [Online]. Available: http://bricklin.com/

[5] Excel Trik. History of Microsoft Excel 1978-2013. [Online]. Available: http://www.exceltrick.com/others/history-of-excel/

[6] H. S. Drier, "Teaching and learning mathematics with interactive spreadsheets," School Science and Mathematics, vol. 101, no. 4, pp. 170-179, 2001.

[7] D. Arganbright, "Enhancing mathematical graphical displays in excel through animation," Spreadsheets in Education (eJSiE), vol. 2, no. 1, article 8 .

[8] R. A. Neurath and L. J. Stephens, "The effect of using Microsoft Excel in a high school algebra class," International Journal of Mathematical Education in Science and Technology, vol. 37, no. 6, pp. 721-726, 2006.

[9] D. Ben-Zvi, "Toward understanding the role of technological tools in statistical learning," Mathematical Thinking and Learning, vol. 2, no. 1-2, pp. 127-155, 2000.

[10] P. C. Bell, "Teaching business statistics with Microsoft Excel," Informs Transactions on Education, vol. 1, no. 1, pp. 18-26, 2000.

[11] K. Jones, "Using spreadsheets in the teaching and learning of mathematics: a research bibliography," MicroMath, vol. 21, no. 1, pp. 30-31, 2005

[12] M. L. Niess, "Scaffolding math learning with spreadsheets. learning connections - mathematics," Learning \& Leading with Technology, vol. 32 , no. 5 , pp. $24-25,2005$.

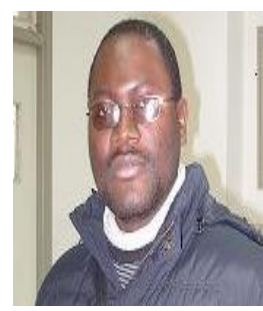

Nchimunya Chaamwe was born in Choma, Zambia, in 1974. He received his BSc in mathematics at University of Zambia, in 2001; and the MSc in information technology systems at the University of Strathclyde in Glasgow Scotland, UK in 2003; and the $\mathrm{PhD}$ in information and communication engineering at Huazhong University of Science and Technology (HUST) in China in 2011.

From 2003 to 2013, he worked as a lecturer in the Copperbelt University in Zambia. From 2007 to 2008, he worked as a assistand dean of school with the same affiliation. And from 2013 to now, he worked as a senior lecturer. He has been published many articles, such as "Mathematical analysis (Algebra, calculus and trigonometry)," Nandanam Publishing House, etc.

Dr. Nhimunya Chaamwe is a member of the Computer Society of Zambia. He has served as a reviewer for the many conferences and journals, such as SAICSIT 2010, Journal of Engineering and Computer innovations and so on.

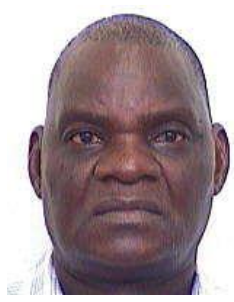

Langstone Shumba was born in Mberengwa district in the Midlands province, Zimbabwe, on April 26, 1955.

He got the Certificate in PC maintenance from July to August 2006. And from October 1987 to February 29, 1988, he studied in University of Zimbabw and received the Certificate in Computer Engineering of Maintenance. Then, he continued his study in Control Data Institute, in London, UK. Later, he got his Msc in computer science engineering degree from Vinnitsa Polytechnic Institute (USSR). He is currently employed by the Copperbelt University (Kitwe, Zambia), as a lecturer in the Computer Science Department.

Mr Shumba's research interests are in computer science of computer security, computer networking and computer architecture. He is a member of the Computer Society of Zimbabwe. 